\title{
The additive role of dynamic contrast- enhanced and diffusion-weighted MR imaging in preoperative staging of breast cancer
}

\author{
Lamiaa Mohamed Bassam Hashem ${ }^{1,2^{*}}$ (D), Yasmine Ahmed Elsayed Sawy ${ }^{1}$, Rasha Mohamed Kamal ${ }^{1,2}$, \\ Soha Mohamed Ahmed ${ }^{3}$ and Dalia Salaheldin elmesidy ${ }^{1,2}$
}

\begin{abstract}
Background: In women with diagnosed breast cancer, accurate loco-regional staging and preoperative examination are of utmost importance for optimal patient management decisions. MRI may be warranted for correct preoperative staging as recommended from international guidelines. DWI-MRI can be combined with CEMRI to assess more functional data. So we aimed to evaluate the performance of CE-MRI and qualitative DWI-MRI in preoperative loco-regional staging of malignant breast lesions as regards the local extension of the disease and axillary lymph node status, beyond standard assessment with mammography and ultrasound. This prospective study included 50 female patients with pathologically proven malignant breast lesions (BIRADS VI) coming for preoperative staging. Full-field digital mammography (FFDM) and ultrasound, CE-MRI, and DWI-MRI findings were compared for all patients, and the findings were evaluated independently. Results were then correlated to postoperative histopathology.
\end{abstract}

Results: Fifty women with pathologically proven malignant breast lesions (BIRADS VI) were enrolled in this study; the mean age of this study population was 43.25 years. The 50 patients were divided into 2 groups: 37/50 (74\%) underwent upfront surgery and 13/50 (26\%) received neoadjuvant therapy before surgery. All patients performed DCE and DWI-MRI breast. Among patients who underwent upfront surgery, DCE-MRI showed the highest correlation with the postoperative pathology size and the overall sensitivity regarding multiplicity. Regarding patients who received neoadjuvant therapy, DCE-MRI was found to have the highest correlation with the postoperative pathology concerning lesion size and multiplicity after completion of the neoadjuvant chemotherapy cycles.

Conclusion: CE-MRI can accurately map lesion extension and detect multifocality/multicentricity, thus tailor surgical management options (either conservative surgery or mastectomy). Qualitative DWI can be combined with ultrasonography for better evaluation of the axillary nodal status.

Keywords: Dynamic contrast-enhanced MRI (DCE-MRI), Diffusion-weighted MRI (DWI-MRI), Mammography, Ultrasound, Malignant breast lesions, Preoperative staging

\footnotetext{
* Correspondence: lamia.bassam22@gmail.com

'Department of Diagnostic and Interventional Radiology, Faculty of

Medicine, Cairo University, Cairo, Egypt

${ }^{2}$ Department of Diagnostic and Interventional Radiology, Baheya Foundation,

Cairo, Egypt

Full list of author information is available at the end of the article
}

\section{Springer Open}

(c) The Author(s). 2021 Open Access This article is licensed under a Creative Commons Attribution 4.0 International License, which permits use, sharing, adaptation, distribution and reproduction in any medium or format, as long as you give appropriate credit to the original author(s) and the source, provide a link to the Creative Commons licence, and indicate if changes were made. The images or other third party material in this article are included in the article's Creative Commons licence, unless indicated otherwise in a credit line to the material. If material is not included in the article's Creative Commons licence and your intended use is not permitted by statutory regulation or exceeds the permitted use, you will need to obtain permission directly from the copyright holder. To view a copy of this licence, visit http://creativecommons.org/licenses/by/4.0/. 


\section{Background}

In women with diagnosed breast cancer, accurate locoregional staging and preoperative examination of the whole breast and both axillae are of utmost importance for optimal patient management decisions and correct choice of therapy [1].

The choice between mastectomy and breastconserving surgery with or without axillary evacuation and with or without neoadjuvant chemotherapy depends on numerous factors. These factors include tumor location, grade and size in relation to breast volume, multifocality or multicentricity, and axillary lymph node status [1]. Overestimation can lead to unnecessary mastectomies and unnecessary axillary evacuation. On the other hand, underestimation of tumor extent may lead to reoperations or recurrence [2].

Clinical examination and conventional imaging (mammography and ultrasound) may underestimate tumor size and/or miss small foci in comparison with histopathological examination. Breast MRI with sensitivity close to $100 \%$ may be warranted for correct preoperative staging as recommended from international guidelines [2]. It assesses the real extension of cancer by evaluating the index lesion dimensions along with identifying additional malignant foci in the same or contralateral breast, thus tailoring surgery options for each patient [3].

Regarding lymph node evaluation, mammography is typically suboptimal [4]. Non-invasive techniques such as ultrasonography (US) and PET-CT have been suggested for the evaluation of the axilla. MRI has some advantages over other modalities such as having low intra and interobserver variation (compared to US) and not utilizing radiation (compared to PET) [5].

Additional MRI parameters such as DWI-MRI can be combined with DCE-MRI to assess more functional data and to overcome limitations in DCE-MRI specificity [6].

So we aimed to evaluate the performance of CE-MRI and qualitative DWI-MRI in preoperative loco-regional staging of malignant breast lesions as regards the local extension of the disease and axillary lymph node status, beyond standard assessment with mammography and ultrasound.

\section{Patients and methods \\ Patients}

This prospective study was conducted over a period of 1 year starting March 2017 till March 2018. The study included 50 women discussed at the MDT with pathologically proven breast cancer (BIRADS VI) who were candidates for further preoperative staging (Fig. 1). The age of patients ranged from 27 to 70 years (mean age $43.25 \pm 9.04 \mathrm{SD}$ ). They were

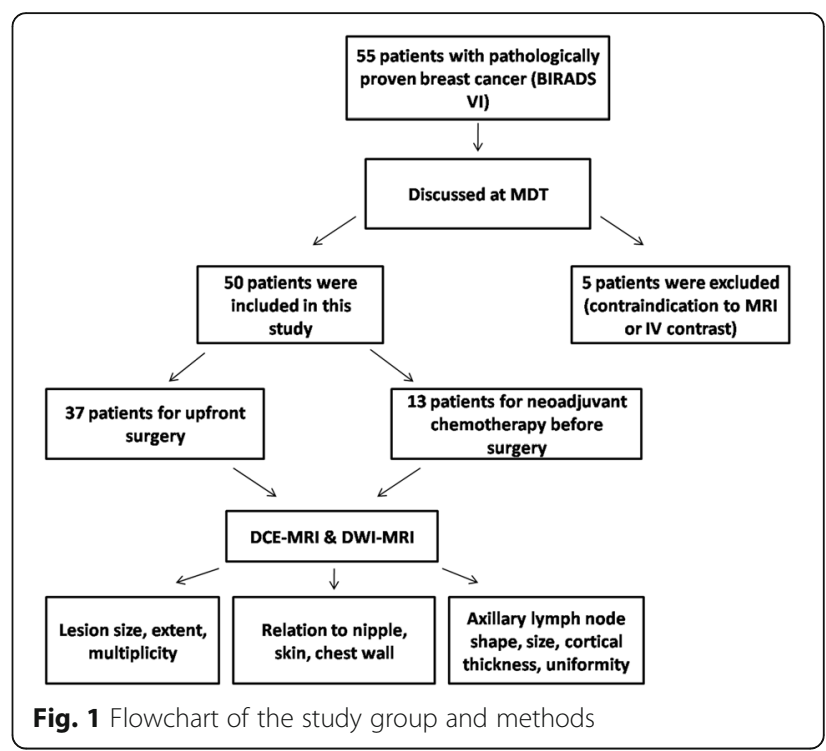

divided into 2 groups: patients who underwent upfront surgery $(n=37 / 50,74 \%)$ and those who received neoadjuvant therapy ( $n=13 / 50,26 \%)$.

Exclusion criteria included patients who have contraindication to MRI (cardiac pacemaker, aneurismal clips, bone growth stimulators) and IV contrast (renal impairment, allergic patients, or those known to have history of anaphylactic reaction from contrast media).

\section{Methods}

All patients $(n=50 / 50)$ had previously reported sonomammography and underwent dynamic MRI with DWIs.

\section{Technique of DCE and DWI-MRI}

MRI was performed using (Philips) $1.5 \mathrm{~T}$ MRI system. The examination was performed using a bilateral breast surface coil with the patient in the prone position. The imaging studies included (1) localizer: axial T1-weighted turbo spin echo $(\mathrm{TR} / \mathrm{TE}=307 / 4.6 \mathrm{~ms})$, axial T2-wighted turbo spin echo $(\mathrm{TR} / \mathrm{TE}=4.3 \mathrm{~s} / 91 \mathrm{~ms})$, and axial shorttime inversion recovery (STIR) $(\mathrm{TR} / \mathrm{TE}=5.2 \mathrm{~s} / 71 \mathrm{~ms}$; inversion time $=170 \mathrm{~ms}$ ) as well as sagittal turbo spin echo weighted sequences; (2) pre-contrast series: seven dynamic acquisitions, one before and one after intravenous injection $0.1 \mathrm{mmol} / \mathrm{kg}$ body weight of contrast material (gadolinium-diethylene tri amino penta acid; Gd-DTPA), using the dynamic THRIVE sequence (T1 high-resolution isotropic volumetric examination) (TR/ $\mathrm{TE}=5 / 2 \mathrm{~ms}$ ); (3) post-contrast series: seven dynamic acquisitions, one before and one after intravenous injection $0.1 \mathrm{mmol} / \mathrm{kg}$ body weight of contrast material (gadolinium-diethylene tri amino penta acid; Gd-DTPA), using the dynamic THRIVE sequence (T1 high-resolution 
Table 1 Correlations between lesion size measured by different imaging modalities and postoperative pathology results in upfront surgery group

\begin{tabular}{lllll}
\hline & Lesions size by different imaging modalities & Postoperative pathology lesions size & Correlation coefficient & $P$ value \\
\hline SM & $2.61 \pm 2.06$ & $3.14 \pm 2.40$ & 0.510 & 0.001 \\
DCE-MRI & $3.73 \pm 2.64$ & $3.14 \pm 2.40$ & 0.858 & $<0.001$ \\
MRI-DWI & $2.46 \pm 2.93$ & $3.14 \pm 2.40$ & 0.322 & 0.052 \\
\hline
\end{tabular}

isotropic volumetric examination); and (4) $D W-M R I$ was performed using single-shot echo planar imaging and fat suppression with repetition time/echo time of 5000 $\mathrm{msec} / 75 \mathrm{msec}, 5-\mathrm{mm}$-section thickness, $30 \times 30 \mathrm{~cm}$ field of view, $256 \times 256,3$-mm-section gap, and 7:14 s acquisition time.

\section{Image analysis}

Image analysis and interpretation were done by a singleconsultant radiologist with more than 10 years of experience in advanced breast imaging techniques, guided by the results of clinical data but blind to the sonomammography findings and the postoperative pathologic diagnosis.

\section{Image analysis and interpretation of MRI}

We characterized each malignant (or additional suspicious) lesion in reference to MRI BIRADS atlas 2013 morphology descriptors [7]. Any detected lesion was assessed for the size, extent, and multiplicity, as well as relation to the skin, nipple, and chest wall with kinetic assessment done. Axillary lymph nodes were also assessed for their shape, size, cortical thickness, and uniformity. The presence or absence of diffusion restriction was mentioned according to the qualitative assessment (i.e., the signal pattern in DWI and ADC map) [8].

The final $\mathrm{T}$ and $\mathrm{N}$ stages of each patient were estimated by sonomammography and MRI according to the AJCC guidelines of breast cancer staging [9]. Regarding the $(\mathrm{T})$ stage, in case of multifocal disease, the overall area was measured, while in case of multicentricity, the size of the largest lesion was used, and in cases of neoadjuvant group, the post-neoadjuvant residual lesion size was the one used for correlation with the final postoperative pathology specimen.

\section{Statistical analysis}

Data were coded and entered using the statistical package SPSS (Statistical Package for the Social Sciences) version 25. Data was summarized using the mean, standard deviation, median, minimum, and maximum in quantitative data and using frequency (count) and relative frequency (percentage) for categorical data. Standard diagnostic indices including sensitivity, specificity, positive predictive value (PPV), negative predictive value (NPV), and diagnostic efficacy were calculated as described by [10]. Correlations between quantitative variables were done using Spearman correlation coefficient [11]. $P$ value less than 0.05 was considered as a statistically significant.

\section{Results}

The study included 50 cases with pathologically proven malignant breast lesions (BIRADS VI). They were divided into 2 groups: patients who underwent upfront surgery $(37 / 50,74 \%)$ and those who had received neoadjuvant chemotherapy before surgery $(13 / 50,26 \%)$.

\section{Group 1: upfront surgery}

The ages ranged from 27 to 70 years with a mean age $43.25 \pm 9.04$ (mean=/-SD). The histopathological types
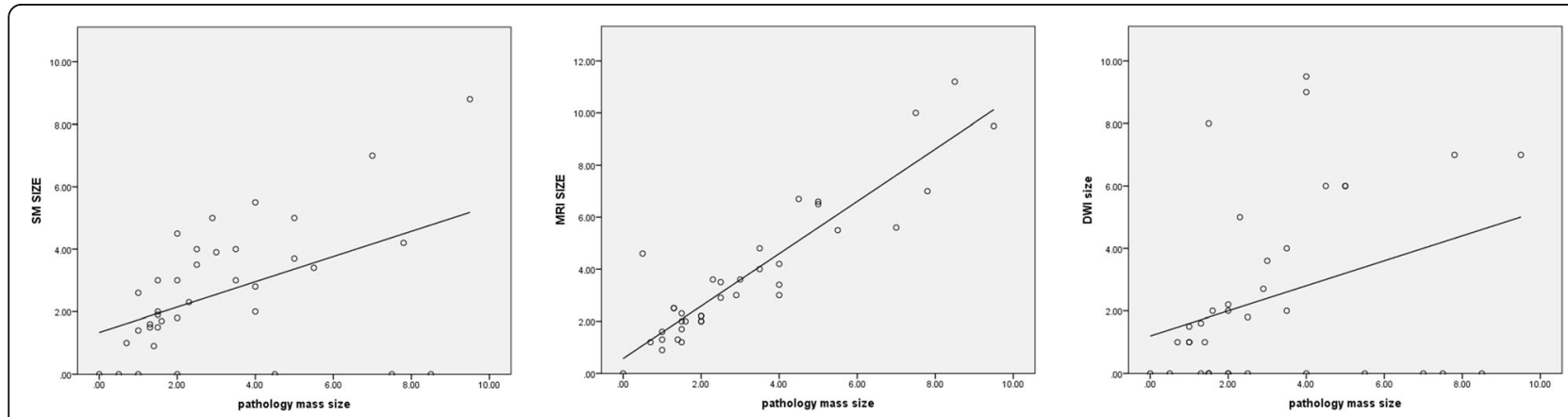

Fig. 2 Scatter dot figure showing the relation between the measured lesion size in different imaging modalities and the postoperative pathology lesion size 
of malignant lesions in this group were 5/37 (13.5\%) DCIS, 8/37 (21.6\%) IDC, 17/37 (45.9\%) IDC/DCIS, 4/37 (10.8\%) ILC, and 1/37 (2.7\%) ILC/IDC, ILC/LCIS, mucinous carcinoma, and tubular/cribriform carcinoma/ DCIS. While the molecular subtypes were 20/37 (54\%) luminal A, 12/37 (32.5\%) luminal B, and 5/37 (13.5\%) triple negative.

The mean size of the malignant mass lesions was measured by ultrasound, DCE-MRI, and DWI-MRI, and the sizes were correlated with the postoperative pathology size (Table 1). A statistically significant correlation was found between the sizes of the lesions measured by dynamic MRI and ultrasound ( $p$ values $<0.001$ and 0.001 , respectively), yet a higher correlation $(R$ 0.858) was calculated for dynamic MRI as seen in the scatter dot (Fig. 2).

Regarding lesion multiplicity, additional lesions were seen by SM and DCE-MRI in 15 and 17 cases, respectively (5 and $9 \mathrm{TP}$, respectively), when only 12 cases were confirmed by the postoperative pathology and DCE-RI showed higher sensitivity. DWI-MRI was found to be unreliable in the assessment of lesion multiplicity (Table 2).

According to the postoperative specimen pathology, 15 cases showed pathological axillary lymph nodes. The axillary lymph node status was overestimated on ultrasound and MRI; 16 on US (11 TP and 5 FP) and 19 on CE-MRI (12 TP and 7 FP). On the other hand, the lymph node status was underestimated by DWIs, and only 13 nodes were reported as pathological (9 $\mathrm{TP}$ and $4 \mathrm{FP}$ ). CE-MRI and ultrasound identified an additional pathological infraclavicular LN that was missed on DWI-MRI. DWI detected pathological axillary LN not detected by CE-MRI. Results are shown in Table 2.

Comparison of diagnostic indices of sonomammography, DCE-MRI, and DWI-MRI regarding lymph node status and multiplicity of malignant lesions is shown in Table 3. The accuracy measures of sonomammography were found to be the highest among the imaging modalities used to assess axillary LNs. On the other hand, regarding multiplicity, the accuracy measures of CE-MRI were found to be higher than those of sonomammography.

The $\mathrm{T}$ and $\mathrm{N}$ stages of the cases in this group are shown in Table 4.

\section{Assessment of the relation to the skin and nipple}

There was no skin invasion detected on both SM and CE-MRI while the nipple was involved in 2 cases by SM and in 6 cases by CE-MRI. In the postoperative pathology, 2 cases showed skin invasion and one case showed nipple affection. DWI-MRI was found to be
Table 2 Table showing multiplicity and axillary lymph nodes status by the different imaging modalities in upfront surgery group

\begin{tabular}{llll}
\hline Multiplicity & Multiplicity & Count & $\%$ \\
Sono-mammography & Single & $15 / 37$ & $40.5 \%$ \\
& Multiple & $15 / 37$ & $40.5 \%$ \\
No & $7 / 37$ & $18.9 \%$ \\
DCE-MRI & Single & $19 / 37$ & $51.4 \%$ \\
& Multiple & $17 / 37$ & $45.9 \%$ \\
Postoperative pathology & No & $1 / 37$ & $2.7 \%$ \\
& Single & $24 / 37$ & $64.9 \%$ \\
Lymph nodes & Multiple & $12 / 37$ & $32.4 \%$ \\
SM AXLNs & No & $1 / 37$ & $2.7 \%$ \\
SM other LNs & LN status & Count & $\%$ \\
& Pathological & $16 / 37$ & $43.2 \%$ \\
DCE-MRI AXLNs & Non specific & $21 / 37$ & $56.8 \%$ \\
& Pathological & $1 / 37$ & $2.7 \%$ \\
Postoperative pathology AXLNs & Pathological & $15 / 37$ & $40.5 \%$ \\
& Non specific & $22 / 37$ & $59.4 \%$ \\
\hline DCE-MRI other LNs & Pathological & $19 / 37$ & $51.4 \%$ \\
& Non specific & $18 / 37$ & $48.6 \%$ \\
& Pathological & $1 / 37$ & $2.7 \%$ \\
& IMLNs & $4 / 37$ & $10.8 \%$ \\
& Pathological & $13 / 37$ & $35.1 \%$ \\
& Non specific & $24 / 37$ & $64.9 \%$ \\
& & & \\
& & $1 / 37$ & $2.7 \%$ \\
& & &
\end{tabular}

unreliable in the estimation of the skin and nipple involvement due to its poor resolution.

\section{Other findings}

Suspicious microcalcifications were seen in 4 cases on the mammography with the corresponding area of nonmass enhancement in 3 of them on DCE-MRI. The 4 cases had corresponding area of DCIS in the postoperative pathology.

\section{Group 2: neoadjuvant chemotherapy group}

The ages ranged from 31 to 60 years with a mean age $42.31 \pm 9.30 \quad$ (mean=/-SD). The histopathological types of malignant lesions in this group were $1 / 13$ (7.7\%) IDC/ILC/DCIS, 2/13 (15.4\%) IDC/DCIS, 9/13 (69.2\%) IDC, and $1 / 13(7.7 \%)$ DCIS. While the molecular subtypes were $1 / 13$ (7.7\%) HER 2+ve, 3/13 (23.1\%) luminal A, 4/13 (30.8\%) luminal B, and 5/13 $(38.5 \%)$ triple negative.

The mean size of the malignant mass lesions postneoadjuvant therapy was measured by ultrasound, DCEMRI, and DWI, and the sizes were correlated with the postoperative pathology size (Table 5). A statistically 
Table 3 Comparison of diagnostic indices of Sonomammography, DCE-MRI and DWI-MRI regarding multiplicity and axillary lymph nodes status in the upfront surgery group

\begin{tabular}{|c|c|c|c|c|c|}
\hline Multiplicity & Sensitivity & Specificity & PPV & NPV & Accuracy \\
\hline SM & $\begin{array}{c}33.33 \% \\
95 \% \mathrm{Cl}: 11.8 \%-61.6 \%\end{array}$ & $\begin{array}{l}90.91 \% \\
95 \% \mathrm{Cl}: 70.8 \%-98.8 \%\end{array}$ & $\begin{array}{l}71.43 \% \\
95 \% \mathrm{Cl}: 35.7 \%-91.8 \%\end{array}$ & $\begin{array}{l}66.67 \% \\
95 \% \mathrm{Cl}: 57.7 \%-74.5 \%\end{array}$ & $\begin{array}{l}67.57 \% \\
95 \% \mathrm{Cl}: 50.2 \%-81.9 \%\end{array}$ \\
\hline DCE-MRI & $\begin{array}{l}81.82 \% \\
95 \% \mathrm{Cl}: 48.2 \%-97.7 \%\end{array}$ & $\begin{array}{l}84.62 \% \\
95 \% \mathrm{Cl}: 63.1 \%-95.6 \%\end{array}$ & $\begin{array}{l}69.23 \% \\
95 \% C l: 46.6 \%-85.2 \%\end{array}$ & $\begin{array}{l}91.67 \% \\
95 \% C l: 75.6 \%-97.5 \%\end{array}$ & $\begin{array}{l}83.78 \% \\
95 \% \mathrm{Cl}: 67.9 \%-93.8 \%\end{array}$ \\
\hline Lymph nodes & Sensitivity & Specificity & PPV & NPV & Accuracy \\
\hline SM & $\begin{array}{c}73.33 \% \\
95 \% \mathrm{Cl}: 44.9 \%-92.2 \%\end{array}$ & $\begin{array}{l}77.27 \% \\
95 \% C l: 54.6 \%-92.1 \%\end{array}$ & $\begin{array}{l}68.75 \% \\
95 \% C l: 48.9 \%-83.4 \%\end{array}$ & $\begin{array}{l}80.95 \% \\
95 \% C l: 64.0 \%-91.02 \%\end{array}$ & $\begin{array}{l}75.68 \% \\
95 \% \text { Cl:58.8\%-88.2\% }\end{array}$ \\
\hline DCE-MRI & $\begin{array}{l}80.00 \% \\
95 \% \mathrm{Cl}: 51.9 \%-95.6 \%\end{array}$ & $68.18 \% 95 \% \mathrm{Cl}: 45.1 \%-86.1 \%$ & $\begin{array}{l}63.16 \% \\
95 \% C l: 46.93 \%-76.8 \%\end{array}$ & $\begin{array}{l}83.33 \% \\
95 \% C l: 63.5 \%-93.4 \%\end{array}$ & $\begin{array}{l}72.97 \% \\
95 \% \text { Cl:55.8\%-86.2\% }\end{array}$ \\
\hline DWI-MRI & $\begin{array}{l}60.00 \% \\
95 \% C l: 32.2 \%-83.6 \%\end{array}$ & $\begin{array}{l}81.82 \% \\
95 \% C l: 59.7 \%-94.8 \%\end{array}$ & $\begin{array}{l}69.23 \% \\
95 \% \mathrm{Cl}: 45.8 \%-85.6 \%\end{array}$ & $\begin{array}{l}75 \% \\
95 \% C l: 61.0 \%-85.1 \%\end{array}$ & $\begin{array}{l}72.97 \% \\
95 \% \mathrm{Cl}: 55.8 \%-86.2 \%\end{array}$ \\
\hline
\end{tabular}

significant correlation was found between the sizes of the lesions measured by dynamic MRI and ultrasound ( $p$ values 0.013 and 0.114 , respectively); yet, a higher correlation $(R$ 0.667) was calculated for dynamic MRI.

The RECIST 1.1 grade of the post-neoadjuvant malignant lesions detected by sono-mammography were $3 / 13$ (23\%) complete response, 5/13 (38.5\%) stable disease, and 5/13 (38.5\%) regressive disease, while those detected by CE-MRI were 7/13 (53.8\%) complete response, $4 / 13(30.8 \%)$ stable disease, and 2/ 13 (15.3\%) regressive disease. And finally, those detected by DWI-MRI were $8 / 13(61.5 \%)$ complete response, $2 / 13(15.3 \%)$ stable disease, and 3/13 (23\%) regressive disease.

Regarding lesion multiplicity, additional lesions detected by SM and DCE-MRI correctly correlated with the postoperative pathology specimen results $(n=3 / 13$ (23.1\%)). DWI-MRI was found to be unreliable in the assessment of lesion multiplicity (Table 6).
Regarding post-neoadjuvant chemotherapy axillary lymph node status, sonomammography and DCEMRI showed similar results (5 cases with pathological LNs) that were identical to those identified in the postoperative pathology. In DWI-MRI, pathological LNs were found in 4 cases only. Regarding additional pathological LNs, 2 additional pathological infraclavicular and supraclavicular LNs were detected by both ultrasound and DCE-MRI, upgrading the $\mathrm{N}$ stage of those cases. Both cases were missed by DWI-MRI (Table 6).

The $\mathrm{T}$ and $\mathrm{N}$ stages of the cases in this group are shown in Table 7.

\section{Discussion}

An accurate loco-regional staging and preoperative assessment of breast cancer are considered of key importance to guide treatment decisions [12]. MRI is a valuable technique and has been increasingly used particularly in

Table 4 Final $\mathrm{T}$ and $\mathrm{N}$ stages of the cases in the upfront surgery group detected by different imaging modalities

\begin{tabular}{|c|c|c|c|c|}
\hline Size $(\mathrm{T})$ & SM & DCE-MRI & MRI-DWI & Pathology \\
\hline Tx & $3 / 37(8.1 \%)$ & $0 / 37$ & $0 / 37$ & $0 / 37$ \\
\hline$\underline{\text { T0 }}$ & 4/37 (10.8\%) & $1 / 37(2.7 \%)$ & 14/37 (37.8\%) & $1 / 37(2.7 \%)$ \\
\hline$\underline{\mathrm{T} 1}$ & 9/37 (24.3\%) & 7/37 (18.9\%) & 7/37 (18.9\%) & 13/37 (35.1\%) \\
\hline$\underline{\mathrm{T}} 2$ & 18/37 (48.6\%) & 20/37 (54\%) & 8/37 (21.6\%) & $16 / 37(43.2 \%)$ \\
\hline$\underline{\mathrm{T} 3}$ & 3/37 (8.1\%) & 9/37 (24.3\%) & 8/37 (21.6\%) & 6/37 (16.2\%) \\
\hline$\underline{\mathrm{T} 4}$ & $0 / 37$ & $0 / 37$ & $0 / 37$ & $1 / 37(2.7 \%)$ \\
\hline Lymph node (N) & SM & DCE-MRI & MRI-DWI & Pathology \\
\hline$N x$ & $3 / 37(8.1 \%)$ & $8 / 37(21.6 \%)$ & $0 / 37$ & $0 / 37$ \\
\hline No & 22/37 (59.4\%) & $17 / 37$ (45.9\%) & $24 / 37(64.8 \%)$ & 22/37 (59.4\%) \\
\hline N1 & 11/37 (29.7\%) & 10/37 (27\%) & $11 / 37$ (29.7\%) & 11/37 (29.7\%) \\
\hline N2 & $1 / 37(2.7 \%)$ & $1 / 37(2.7 \%)$ & $1 / 37(2.7 \%)$ & $3 / 37(8.1 \%)$ \\
\hline N3 & $0 / 37$ & $1 / 37(2.7 \%)$ & $1 / 37(2.7 \%)$ & $1 / 37(2.7 \%)$ \\
\hline
\end{tabular}


Table 5 Correlations between lesion size measured by different imaging modalities and postoperative pathology result in the neoadjuvant goup

\begin{tabular}{lllll}
\hline & Post-neoadjuvant chemotherapy lesion size & Postoperative pathology lesion size & Correlation coefficient & $P$ value \\
\hline SM & $1.71 \pm 1.23$ & $1.40 \pm 1.77$ & 0.460 & 0.114 \\
DCE-MRI & $1.21 \pm 1.64$ & $1.40 \pm 1.77$ & 0.667 & 0.013 \\
MRI-DWI & $1.23 \pm 1.79$ & $1.40 \pm 1.77$ & 0.226 & 0.459 \\
\hline
\end{tabular}

cases in which sonomammography are inconclusive or yield discrepancies (Fig. 3). It may improve the analysis of the local extent of breast cancer [13], thus increasing the rates of complete resection and reducing the number of re-operations [14].

Therefore, in this study, we aimed to evaluate the performance of CE-MRI and qualitative DWI-MRI in preoperative loco-regional staging of malignant breast lesions as regards the local extension of the disease and axillary lymph node status, beyond standard assessment with mammography and ultrasound.

We divided our cases in this study into 2 groups: patients who underwent upfront surgery $(37 / 50,74 \%)$ and those who had received neoadjuvant chemotherapy before surgery $(13 / 50,26 \%)$.

We started the analysis of the upfront surgery group by correlating the size of the malignant lesion with the postoperative pathology results. CE-MRI showed the highest correlation while DWI showed the lowest (Fig. 4).

In a study performed by Gundry, tumor size was underestimated on both mammography and ultrasound (14 and $18 \%$, respectively); however, the size of the tumor at histology was not significantly different from that seen on MRI [15].

In our study, CE-MRI was able to detect the DCIS component of the malignant lesions in the form of area of non-mass enhancement giving better extent of the tumor size and consequently superior staging results over the sonomammography.

Kim et al. also stated that MRI was more accurate compared to mammography in the assessment of the DCIS size [16]. According to Hwang et al., MRI was

Table 6 Table showing multiplicity and axillary lymph nodes status by the different imaging modalities in neo-adjuvant group

\begin{tabular}{|c|c|c|c|}
\hline Multiplicity & Multiplicity & Count & $\%$ \\
\hline \multirow[t]{3}{*}{ SM post chemotherapy } & Single & $7 / 13$ & $53.8 \%$ \\
\hline & Multiple & $3 / 13$ & $23.1 \%$ \\
\hline & No & $3 / 13$ & $23.1 \%$ \\
\hline \multirow[t]{3}{*}{ DCE-MRI post chemotherapy } & Single & $6 / 13$ & $46.2 \%$ \\
\hline & Multiple & $3 / 13$ & $23.1 \%$ \\
\hline & No & $4 / 13$ & $30.8 \%$ \\
\hline \multirow[t]{2}{*}{ Postoperative pathology } & Single & $3 / 13$ & $23.1 \%$ \\
\hline & Multiple & $3 / 13$ & $23.1 \%$ \\
\hline Lymph nodes & LN status & Count & $\%$ \\
\hline \multirow[t]{2}{*}{ SM post chemotherapy ALN } & Pathological & $5 / 13$ & $30.8 \%$ \\
\hline & Non specific & $8 / 13$ & $61.5 \%$ \\
\hline SM post chemotherapy other LNs & Non specific & $13 / 13$ & $100.0 \%$ \\
\hline \multirow[t]{2}{*}{ DCE-MRI post chemotherapy ALN } & pathological & $5 / 13$ & $38.4 \%$ \\
\hline & Non specific & $8 / 13$ & $61.5 \%$ \\
\hline MRI Post chemotherapy other LNs & Non specific & $13 / 13$ & $100.0 \%$ \\
\hline \multirow[t]{2}{*}{ DWI-MRI post chemotherapy ALN } & pathological & $4 / 13$ & $30.8 \%$ \\
\hline & Non specific & $9 / 13$ & $69.2 \%$ \\
\hline DWI-MRI post chemotherapy other LNs & Non specific & $13 / 13$ & $100.0 \%$ \\
\hline \multirow[t]{2}{*}{ Postoperative pathology } & pathological & $5 / 13$ & $38.4 \%$ \\
\hline & Non specific & $8 / 13$ & $61.5 \%$ \\
\hline
\end{tabular}


Table 7 Final $\mathrm{T}$ and $\mathrm{N}$ stages of the cases in the neoadjuvant group detected by different imaging modalities

\begin{tabular}{|c|c|c|c|c|}
\hline Size (T) & $\underline{S M}$ & DCE-MRI & MRI-DWI & Pathology \\
\hline$\underline{\text { T0 }}$ & $2 / 13(15.3 \%)$ & $7 / 13(53.8 \%)$ & $8 / 13(61.5 \%)$ & $7 / 13(53.8 \%)$ \\
\hline$\underline{\mathrm{T} 1}$ & $5 / 13(38.4 \%)$ & 1/13 (7.7\%) & $0 / 13$ & 1/13 (7.7\%) \\
\hline $\mathrm{T} 2$ & $6 / 13(46.1 \%)$ & $5 / 13(38.4 \%)$ & $5 / 13(38.4 \%)$ & $4 / 13(30.7 \%)$ \\
\hline T3 & $0 / 13$ & $0 / 13$ & $0 / 13$ & $0 / 13$ \\
\hline$\underline{\mathrm{T}} 4$ & $0 / 13$ & $0 / 13$ & $0 / 13$ & 1/13 (7.7\%) \\
\hline Lymph node (N) & $\underline{S M}$ & DCE-MRI & MRI-DWI & Pathology \\
\hline$\underline{\mathrm{Nx}}$ & $1 / 13(7.7 \%)$ & $1 / 13(7.7 \%)$ & $0 / 13$ & $0 / 13$ \\
\hline No & $8 / 13(61.5 \%)$ & $8 / 13(61.5 \%)$ & 9/13 (69.2\%) & $8 / 13(61.5 \%)$ \\
\hline N1 & 4/13 (30.7\%) & 4/13 (30.7\%) & 4/13 (30.7\%) & $2 / 13(15.3 \%)$ \\
\hline N2 & $0 / 13$ & $0 / 13$ & $0 / 13$ & $5 / 13(38.4 \%)$ \\
\hline
\end{tabular}

superior to mammography in the detection of invasive components within DCIS [17].

Regarding lesion multiplicity in our study, the accuracy measures of CE-MRI were found to be higher than those of sonomammography with an overall accuracy of $83.78 \%$ for MRI and $67.57 \%$ for sonomammography with reference to the postoperative pathology results (Fig. 5). DWI-MRI was found to be unreliable due to its poor resolution in the detection of the small satellite lesions.

Additional lesions (multifocal or multicentric disease) have been shown in $31 \%$ of women with breast cancer. Even though mammography and ultrasonography can often detect the primary tumor, small additional lesions may be missed [15].

Selvi et al. stated that MRI is well known for its increased diagnostic value in detecting multifocal, multicentric, or contralateral disease unrecognized on conventional exams [18]. Moreover, the metaanalysis of 50 studies $(10,811$ women with breast cancer) performed by Lee et al. showed that MRI findings prompted conversion from lumpectomy to mastectomy in $12.8 \%$ of cases [4].

Appropriate evaluation of regional lymph node status $(\mathrm{N})$ is important for staging, treatment planning, and prognosis [4]. In reference to the postoperative pathology results of axillary lymph nodes, the accuracy measures of sonomammography were found to be the highest among the imaging modalities, with an overall accuracy of $75.68 \%$ as compared to CEMRI and DWI-MRI with the same accuracy of 72.97\% (Figs. 5 and 6).

The reported axillary US sensitivities, and specificities are in the range of $45.2-100 \%$ and $50-89 \%$, respectively, while those of DCE-MRI are 79-100\% (relatively high) and $56-93 \%$ (relatively low), respectively, and 53.894.7\% and 77-91.7\%, respectively, of DW-MRI for ALN metastases [19].

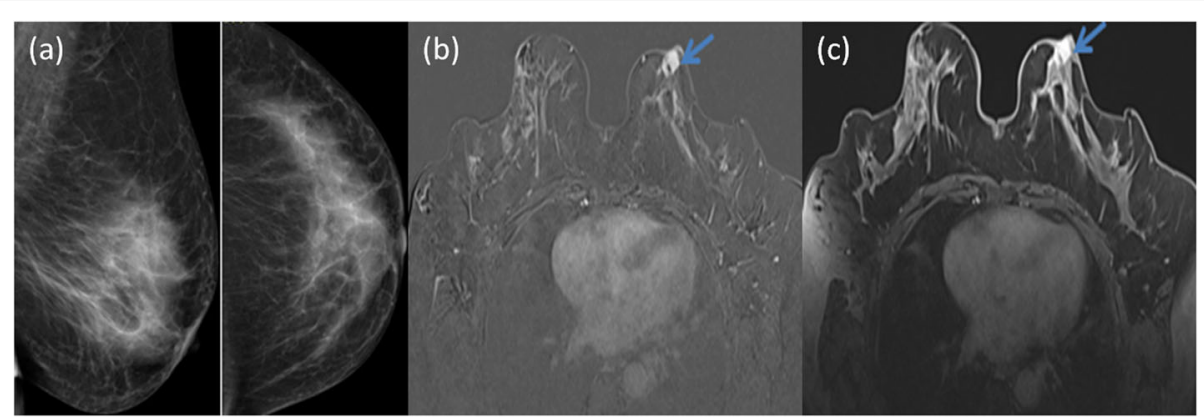

Fig. 3 A case of pathologically proven invasive tubular cribriform carcinoma with skin invasion (T4N0). a Mammgraphy showed no spiculated mass lesions or microcalcific clusters, complementary ultrasound suspected left retroareolar irregular lesion with posterior shadowing related to the nipple. b, c DCE-MRI showed clearly left non-circumscribed ovoid mass related to the posterior aspect of the nipple. It is seen eliciting intense heterogeneous enhancement with central non-enhancing core, facilitated diffusion was noted on DWI-MRI. In this case, the lesion was detected by DCE-MRI and was subtle sonsonography yet missed on mammography and DWI-MRI 


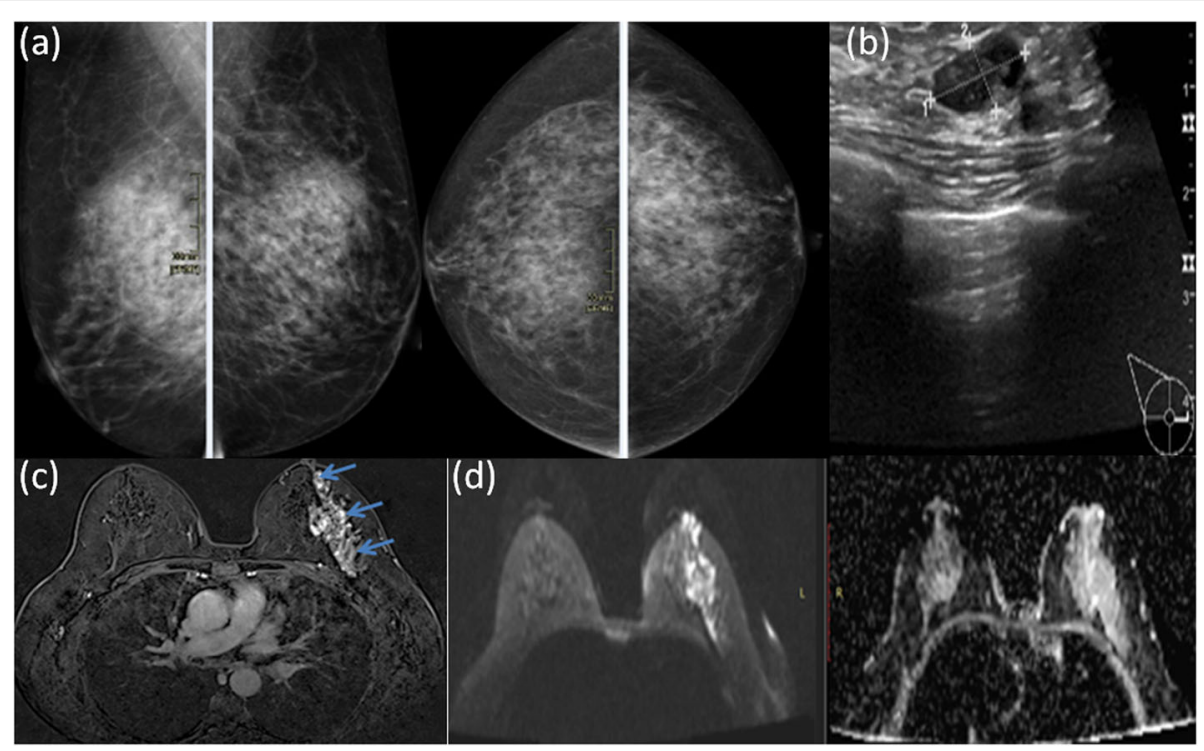

Fig. 4 A case of DCIS with microinvasive carcinoma (T3N1). a Mammography revealed no spiculated mass lesions or microcalcifications. b Complementary US revealed upper central and outer quadrant benign looking lesions; however, biopsy was recommended. c DCE-MRI breast revealed left breast segmental heterogeneous area of pathological non-mass enhancement seen partially involving both the upper and lower outer quadrants extending from 1 to 5 o'clock position. The pathological enhancement is seen extending to reach the nipple. $\mathbf{d}$ Facilitated diffusion was noted on DWI-MRI. In this case, DCE-MRI revealed the same tumor size stage correlating to the pathological specimen result, while SM and DWI-MRI missed the lesion

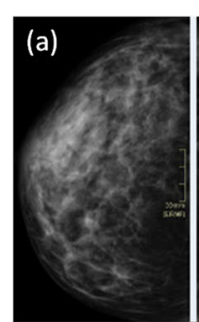

(d) $y$

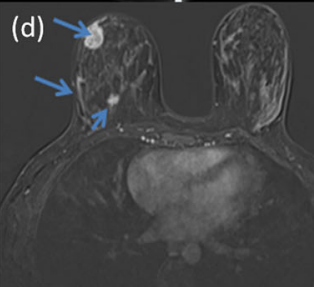

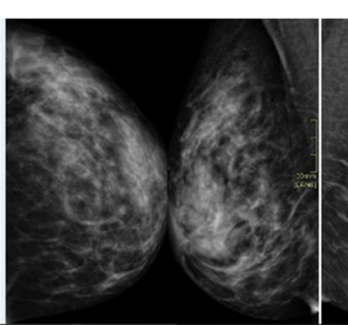
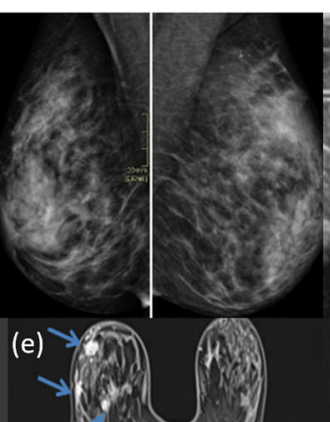

(b)

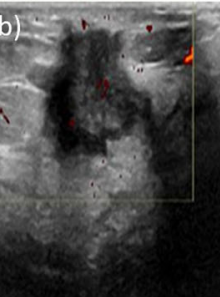

(f)

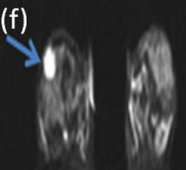

V.
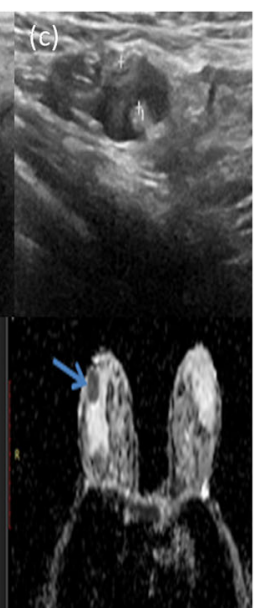

Fig. 5 A case of pathologically proven multicentric IDC with major DCIS component IDC grade II (T2) sentinel lymph node status 1/5(N1). a Mammography showed no spiculated mass lesions or microcalcific clusters. Complementary ultrasound showed b right periareolar 8 o'clock illdefined hypoechoic mass with increased vascularity and $\mathbf{c}$ right pathologically enlarged axillary lymph node. $\mathbf{d}$, e DCE-MRI breast revealed a spiculated heterogeneously enhancing mass at 8 o'clock in the LOQ. The mass is seen reaching the nipple, associated multiple right breast central and lower quadrants similar enhancing nodules are seen. Lower outer quadrant regional pathological non mass enhancement is seen as well. f DWIs showed restricted diffusion. In this case, breast U/S and DWI-MRI detected a single lesion, while DCE-MRI was able to detect multicentricity of the lesions and correlated the best with the pathological specimen results 


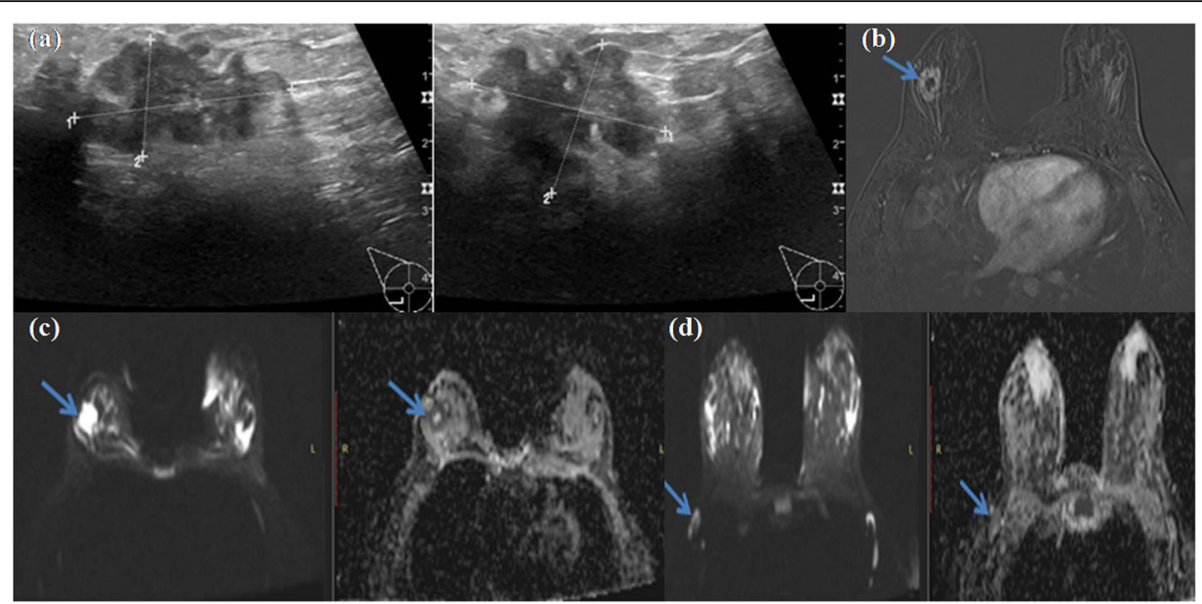

Fig. 6 A case of pathologically proven IDC grade II (T2). a Breast U/S revealed a partially defined irregular shaped hypoechoic mass lesion at right 7-8 o'clock. b DCE-MRI revealed right partially circumscribed ring enhancing breast mass with central breakdown at the lower outer quadrant with restricted diffusion (bright on DWI and dark on ADC with central bright signal (breakdown)). c Right axillary pathologically enlarged LN showing restricted diffusion in the DWI-MRI. $\mathbf{d}$ In this case, the tumor size (T) detected by DCE-MRI and ultrasound were correlating with pathological specimen results, while DWI-MRI was able to detect the pathological axillary LN that was missed by DCE-MRI and SM

In Chung et al. study, the sensitivity, specificity, and accuracy of axillary US and CE-MRI with DWI preoperatively were $100 \%, 83.3 \%$, and $93.6 \%$, respectively. Moreover, DW MRI showed superior diagnostic performance to axillary US [19] (Fig. 6).

According to Lee et al., high-resolution US is useful in the evaluation of lymph nodes at all levels while MRI can in addition detect internal mammary and supraclavicular adenopathy [4].
Kujis et al. also showed in a meta-analysis study done to demonstrate whether MRI can replace SLNB to exclude axillary lymph node metastasis, a false-negative rate of $8.61 \%$ of the SLNB [20].

We then assessed the post-neoadjuvant group. We started the analysis of our cases by correlating the postneoadjuvant therapy size of lesions with the postoperative pathology results. In our study, CE-MRI was found

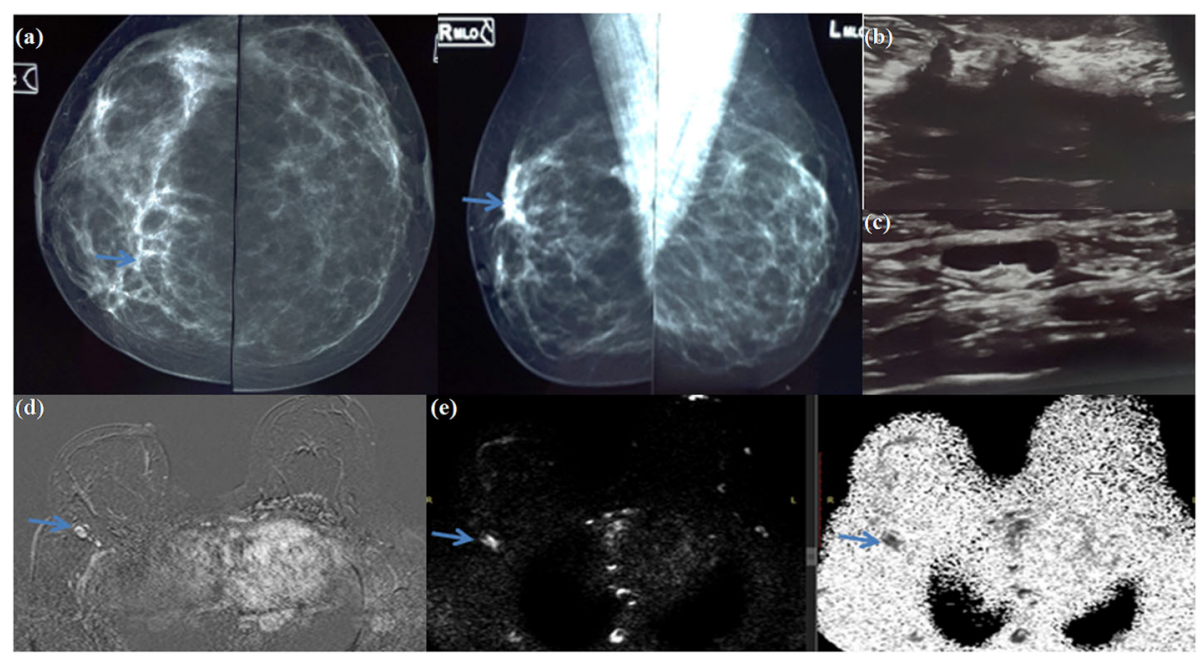

Fig. 7 A case with right breast multicentic malignant lesions, received neoadjuvant chemotherapy with pathologically proven completely burnt out tumor (complete pathological response). a Post-neoadjuvant chemotherapy mammography revealed UIQ architectural distortion. $\mathbf{b}$ Ultrasound showed multiple masses seen extending from right 11 to 2 o'clock position of ill-defined outline and distortion located at 1 o'clock. $\mathbf{c}$ Right indeterminate axillary LN with increased cortical thickness was also seen. $\mathbf{d}$ DCE-MRI showed no sizable gross enhancing masses, yet a pathologically enlarged right axillary lymph node with prominent cortex was noted. e Showing restricted diffusion on the DWI-MRI. In this case, the findings of DCE-MRI and DWI-MRI were correlating with the pathological specimen results regarding the tumor and axillary LNs, while SM gave a false positive result of the mass lesion 
to be the best among the imaging modalities, with a $p$ value of 0.013 (Fig. 7).

According to a study that included 160 patients who received neoadjuvant chemotherapy, the results showed that MRI correlated better with the size of the breast tumor remnants found in the assessment of the surgical specimen than did mammography and ultrasound [14]. Menzes et al. results suggest that DWI and ADC are useful for predicting tumor response to NAC in breast cancer patients [21].

Rosen et al. stated that the treatment options in the neoadjuvant setting depend on the amount of residual tumor. Palpation and conventional imaging (mammography and sonography) have been traditionally used, yet the tumor's true size may be hindered by edema and necrosis at the tumor site [22].

Mammographic assessment becomes more difficult in denser breasts, with ill-defined tumors, or in areas of architectural distortion alone. Sonography may be more accurate with well-defined tumors but may still produce erroneous results [15].

MRI provides anatomic and physiologic evaluation of the tumor, so the findings are not influenced by necrosis and edema. And since the findings are based on the vascularity of the tumor, the effect of chemotherapy agents can be seen through inhibiting tumor angiogenesis [15]. However, some studies emphasize on the tendency of MRI to overestimate lesion size, particularly in women with invasive lobular carcinoma and DCIS [21].

Regarding post-neoadjuvant chemotherapy lymph nodes status in our study, sonomammography and DCE-MRI had the same statistical results, correlating with the postoperative pathology specimen results.

DCE-MRI is correlating better with the postoperative pathology specimen results regarding post-neoadjuvant chemotherapy lesions multiplicity.

MRI prior to NAC is essential especially if there is multicentric or diffuse disease. As a result of therapy, these lesions become less conspicuous with decreased contrast enhancement [15].

According to a study of 163 patients, preoperative MRI showed $91.04 \%$ sensitivity in detecting additional lesions either in the ipsilateral or contralateral breast [18].

Direct skin invasion and involvement of the nipple, pectoralis muscle, or chest wall should be mentioned in radiology reports because their presence changes surgical planning [4].

DCE-MRI and sonomammography correlated well with the postoperative pathology specimen regarding nipple affection while both overestimated the skin involvement. DWI-MRI was found to be unreliable in the estimation of both due to its poor resolution.
According to Gundry, breast MRI can determine chest wall invasion better than mammography or ultrasound. Tumor involvement of the chest wall, regardless of primary tumor size, changes the disease stage to IIIB [15].

We had few limitations in this study, the small number of patients we included, especially in the postneoadjuvant group.

\section{Conclusion}

CE-MRI can accurately map lesion extension and detect multifocality/multicentricity, thus tailor surgical management options (either conservative surgery or mastectomy) for each patient. Qualitative DWI can be combined with ultrasonography for better evaluation of the axillary nodal status of those patients. Combining both allows rather reliable, accurate preoperative locoregional staging of malignant breast disease, avoiding either over or under staging, ensuring that appropriate management is provided for each case.

\section{Abbreviations \\ MRI: Magnetic resonance imaging; US: Ultrasonography; PET-CT: Positron emission tomography computed tomography; DW-MRI: Diffusion weighted magnetic resonance imaging; DCE-MRI: Dynamic contrast enhanced magnetic resonance imaging; CE-MRI: Contrast enhanced magnetic resonance imaging; MDT: Multi-disciplinary team; BIRADS: Breast imaging reporting and data system; DWI and ADC: Diffusion-weighted images and apparent diffusion coefficient; AJCC: American Joint Committee Cancer; DCIS: Ductal carcinoma in situ; IDC: Invasive ductal carcinoma; ILC: Invasive lobular carcinoma; LCIS: Lobular carcinoma in situ; SM: Sonomammography; TP: True positive; FP: False positive; LN: Lymph node; NAC: Neoadjuvant chemotherapy; RECIST: Response evaluation criteria in solid tumors; ALN: Axillary lymph node; SLNB: Sentinel lymph node biopsy}

\section{Acknowledgements}

No Acknowledgements.

\section{Authors' contributions}

DE wrote the manuscript. YS collected patient data. LH worked on image processing and collection of patient's images. RK participated in the design of the study and performed the statistical analysis. YS and LH conceived the study, and participated in its design and coordination and helped to draft the manuscript. RK was responsible of revision of the draft from clinical point of view. SA collected the clinical and pathological data and statistics analysis. All authors have read and approved the manuscript.

\section{Funding}

No funding sources.

\section{Availability of data and materials}

The data sets used and analyzed during the current study are available from the corresponding author on reasonable request.

\section{Ethics approval and consent to participate}

The study was approved by the ethical committee of "Faculty of medicine, Cairo University" with ethical committee approval number and date not available. An informed written consent was taken from all subjects.

\section{Consent for publication}

All patients included in this research gave written informed consent to publish the data contained within this study.

Competing interests

No financial or non-financial competing interests. 


\section{Author details}

${ }^{1}$ Department of Diagnostic and Interventional Radiology, Faculty of Medicine, Cairo University, Cairo, Egypt. ${ }^{2}$ Department of Diagnostic and Interventional Radiology, Baheya Foundation, Cairo, Egypt. ${ }^{3}$ Department of Clinical Oncology, Faculty of Medicine, Cairo University, Cairo, Egypt.

Received: 24 September 2020 Accepted: 7 January 2021

Published online: 25 January 2021

\section{References}

1. Biacchiardi CP, Brizzi D, Genta F et al (2011) Breast cancer preoperative staging: does contrast-enhanced magnetic resonance mammography modify surgery? Int J Breast Cancer:757234. 10 pages. https://doi.org/10. 4061/2011/757234

2. Ahsberg K, Gardfjell A, Nimeus E et al (2020) Added value of contrastenhanced mammography (CEM) in staging of malignant breast lesions-a feasibility study. World J Surg Oncol 18(1):100. https://doi.org/10.1186/ s12957-020-01865-0

3. Bicchierai G, Tonelli P, Piacenti A et al (2020) Evaluation of contrastenhanced digital mammography (CEDM) in the preoperative staging of breast cancer: large-scale single-center experience. Breast J 26(122). https:// doi.org/10.1111/tbj.13766

4. Lee SC, Payal AJ, Samir C et al (2014) Radiologists' role in breast cancer staging: providing key information for clinicians. RadioGraphics 34(2):330342. https://doi.org/10.1148/rg.342135071

5. Arslan G, Altintoprak KM, Yirgin IK et al (2016) Diagnostic accuracy of metastatic axillary lymph nodes in breast MRI. SpringerPlus 5(1):735. https:// doi.org/10.1186/s40064-016-2419-7

6. Gilbert F, Pinker K (2019chapter 13) Diagnosis and staging of breast cancer: when and how to use mammography, tomosynthesis, ultrasound, contrastenhanced mammography, and magnetic resonance imaging. In: Hodler J, Kubik-Huch RA, von Schulthess GK (eds) Diseases of the chest, breast, heart and vessels 2019-2022. Diagnostic and interventional imaging. https://doi. org/10.1007/978-3-030-11149-6_13

7. Morris EA, Comstock CE, Lee $\mathrm{CH}$ et al (2013) ACR BI-RADS ${ }^{\oplus}$ magnetic resonance imaging. In: $\mathrm{ACR} B \mathrm{BI}-\mathrm{RADS}^{\circledR}$ atlas, breast imaging reporting and data system. American College of Radiology, Reston

8. Pereira FP, Martins G, Figueiredo E et al (2009) Assessment of breast lesions with diffusion weighted MRI: comparing the use of different b-values. AJR Am J Roentgenol 193(4):1030-1035. https://doi.org/10.2214/AJR.09.2522

9. Gabriel NH, James LC, Carl JD et al (2017) Breast. In: Mahul BA (ed) American joint committee on cancer (AJCC). AJCC cancer staging manual, 8th edn. Springer, New York, pp 589-628

10. Galen RS (1980) Predictive values and efficiency of laboratory testing. Pediat J Clin North Am 27:861-869. https://doi.org/10.1016/s0031-3955(16)33930-x

11. Chan YH (2003) Biostatistics 104: correlational analysis. Singapore Med 44(12):614-619 PMID: 14770254

12. Lai HW, Chen CJ, Lin YJ et al (2016) Does breast magnetic resonance imaging combined with conventional imaging modalities decrease the rates of surgical margin involvement and reoperation?: a case-control comparative analysis. Medicine (Baltimore) 95(22):e3810. https://doi.org/10. 1097/MD.0000000000003810

13. Pareira FP, Martins G (2011) Diffusion magnetic resonance imaging of the breast. Magn Reson Imaging Clin N Am 19(1):95-110. https://doi.org/10. 1016/j.mric.2010.09.001

14. França $L K$, Bitencourt $A G$, Paiva $H L$ et al. Role of magnetic resonance imaging in the planning of breast cancer treatment strategies: comparison with conventional imaging techniques. Radiol Bras. (2017); 50(2): 76-81. doi: 10.1590/0100-3984.2015.0124.

15. Gundry KR (2005) The application of breast MRI in staging and screening for breast cancer. Oncology (Williston Park) 19:159-169 discussion 170, 173-4, 177. PMID: 15770888

16. Kim DY, Moon WK, Cho $\mathrm{N}$ et al (2007) MRI of the breast for the detection and assessment of the size of ductal carcinoma in situ. Korean J Radiol 8(1): 32-39. https://doi.org/10.3348/kjr.2007.8.1.32

17. Hwang ES, Kinkel K, Esserman LJ et al (2003) Magnetic resonance imaging in patients diagnosed with ductal carcinoma-in-situ: value in the diagnosis of residual disease, occult invasion, and multicentricity. Ann Surg Oncol 10: 381-388. https://doi.org/10.1245/aso.2003.03.085

18. Selvi V, Nori J, Meattini I et al (2018) Role of magnetic resonance imaging in the preoperative staging and work-up of patients affected by invasive lobular carcinoma or invasive ductolobular carcinoma. Biomed Res Int 2018 1569060. https://doi.org/10.1155/2018/1569060

19. Chung J, Youk JH, Kim J et al (2014) Role of diffusion-weighted MRI: predicting axillary lymph node metastases in breast cancer. Acta Radiologica 55(8):909-916. https://doi.org/10.1177/0284185113509094

20. Kuijs VJL, Moossdorff M, Schipper RJ et al (2015) Role of MRI in axillary lymph node imaging in breast cancer patients: a systematic review. Insights Imaging 6(2):203-215. https://doi.org/10.1007/s13244-015-0404-2

21. Menezes GL, Knuttel FM, Stehouwer BL et al (2014) Magnetic resonance imaging in breast cancer: a literature review and future perspectives. World J Clin Oncol 5(2):61-70. https://doi.org/10.5306/wjco.v5.i2.61

22. Rosen EL, Blackwell KL, Baker JA et al (2003) Accuracy of MRI in the detection of residual breast cancer after neoadjuvant chemotherapy. Am J Roentgenol 181(5):1275-1282. https://doi.org/10.2214/ajr.181.5.1811275

\section{Publisher's Note}

Springer Nature remains neutral with regard to jurisdictional claims in published maps and institutional affiliations.

\section{Submit your manuscript to a SpringerOpen ${ }^{\circ}$ journal and benefit from:}

- Convenient online submission

- Rigorous peer review

- Open access: articles freely available online

- High visibility within the field

- Retaining the copyright to your article

Submit your next manuscript at $>$ springeropen.com 